\title{
Solution of a system of differential equations with constant coefficients using inverse moments problem techniques
}

\author{
Dra. María B. Pintarelli * \\ Grupo de Aplicaciones Matemáticas y Estadísticas de la Facultad de Ingeniería, and Departamento de Matemática, Facultad de Cien- \\ cias Exactas ,Universidad Nacional de La Plata, La Plata, Prov. de Buenos Aires, Argentina \\ *Corresponding author E-mail: mariabpintarelli@gmail.com
}

\begin{abstract}
It is known that given a system of simultaneous linear differential equations with constant coefficients you can apply the Laplace method to solve it. The Laplace transforms are found and the problem is reduced to the resolution of an algebraic system of equations of the determining functions, and applying the inverse transformation the generating functions are determined, solutions of the given system. This implies the need to know the analytical form of the inverse transform of the function. In this case the initial conditions consist in knowing the value that the generating function and its derivatives takes at zero. A generalization of this method is proposed in this work, which is to define a more general integral operator than the Laplace transform, the initial conditions consist of Cauchy conditions in the contour. And finally, we find a numerical approximation of the inverse transformation of the generating functions, using the techniques of inverse moment problems, without being necessary to know the analytical form of the inverse transform of the function.
\end{abstract}

Keywords: Integral Equations; Inverse Moment Problem; Laplace Transform; Simultaneous Linear Differential Equations; Solution Stability.

\section{Introduction}

Given a system of ordinary linear differential equations with constant coefficients of the form

$$
\begin{gathered}
y_{1}^{(n)}(x)=f_{1}\left(x, y_{1}, y_{2}, \ldots, y_{k}, y_{1}^{(1)}, \ldots, y_{k}^{(1)}, \ldots, y_{1}^{(n-1)}, \ldots, y_{k}^{(n-1)}\right) \\
\vdots \\
y_{k}^{(n)}(x)=f_{k}\left(x, y_{1}, y_{2}, \ldots, y_{k}, y_{1}^{(1)}, \ldots, y_{k}^{(1)}, \ldots, y_{1}^{(n-1)}, \ldots, y_{k}^{(n-1)}\right)
\end{gathered}
$$

Where $y_{i}^{(n)}(x)$ indicates the derivative of order $n$ of $y_{i}(x)$ $\mathrm{i}=1, \ldots, \mathrm{k}$ and

$$
f_{i}\left(x, y_{1}, y_{2}, \ldots, y_{k}, y_{1}^{(1)}, \ldots, y_{k}^{(1)}, \ldots, y_{1}^{(n-1)}, \ldots, y_{k}^{(n-1)}\right)
$$

$\mathrm{i}=1, \ldots, \mathrm{k}$ are linear functions of $\mathrm{x}, \mathrm{y}_{1}(\mathrm{x}), \mathrm{y}_{2}(\mathrm{x}), \ldots, \mathrm{y}_{\mathrm{k}}(\mathrm{x})$,

$y_{1}^{(1)}(x), \ldots, y_{k}^{(1)}(x), \ldots, y_{1}^{(n-1)}(x), \ldots, y_{k}^{(n-1)}(x)$ with constant coefficients, we want to find the functions $\mathrm{y}_{1}(\mathrm{x}), \mathrm{y}_{2}(\mathrm{x}), \ldots, \mathrm{y}_{\mathrm{k}}(\mathrm{x})$

which are solution of the given system.

There are a variety of methods to solve this problem, exposed in detail in the literature $[1-4]$. Some consist of numerical approximations, others give the exact solution.

If the domain of the unknowns functions is $(0, \infty)$ and are known

$$
\begin{gathered}
\mathrm{y}_{1}(0), \mathrm{y}_{1}^{(1)}(0), \ldots \ldots \ldots, \mathrm{y}_{1}^{(\mathrm{n}-1)}(0) \\
\vdots \\
\mathrm{y}_{\mathrm{k}}(0), \mathrm{y}_{\mathrm{k}}^{(1)}(0), \ldots \ldots \ldots, \mathrm{y}_{\mathrm{k}}^{(\mathrm{n}-1)}(0)
\end{gathered}
$$

a known method [3] is to apply the Laplace transform to each system equation (1). Remember that the Laplace transform is defined as

$$
L(y)=\int_{0}^{\infty} y(x) e^{-\alpha x} d x
$$

And integrating (2) in parts again and again, we come to the well known property

$$
\begin{aligned}
& \mathrm{L}\left(\mathrm{y}^{(\mathrm{n})}(\mathrm{x})\right)= \\
& =\alpha^{\mathrm{n}} \mathrm{L}(\mathrm{y}(\mathrm{x}))-\left(\alpha^{\mathrm{n}-1} \mathrm{y}(0)+\cdots+\alpha \mathrm{y}^{(\mathrm{n}-2)}(0)+\mathrm{y}^{(\mathrm{n}-1)}(0)\right)
\end{aligned}
$$

In this way, applying the Laplace transform to (1) a system of algebraic equations is determined

$$
\begin{array}{ccc}
\mathrm{a}_{11}(\alpha) \mathrm{L}\left(\mathrm{y}_{1}\right)+\mathrm{a}_{12}(\alpha) \mathrm{L}\left(\mathrm{y}_{2}\right)+\ldots & +\mathrm{a}_{1 \mathrm{k}}(\alpha) \mathrm{L}\left(\mathrm{y}_{\mathrm{k}}\right)=\mathrm{c}_{1}(\alpha) \\
\vdots & \ldots & \vdots \\
\mathrm{a}_{\mathrm{k} 1}(\alpha) \mathrm{L}\left(\mathrm{y}_{1}\right)+\mathrm{a}_{\mathrm{k} 2}(\alpha) \mathrm{L}\left(\mathrm{y}_{2}\right)+ & \ldots & +\mathrm{a}_{\mathrm{kk}}(\alpha) \mathrm{L}\left(\mathrm{y}_{\mathrm{k}}\right)=\mathrm{c}_{\mathrm{k}}(\alpha)
\end{array}
$$

Where the unknowns are $\mathrm{L}\left(\mathrm{y}_{1}\right), \mathrm{L}\left(\mathrm{y}_{2}\right), \ldots, \mathrm{L}\left(\mathrm{y}_{\mathrm{k}}\right)$.

When solving this system, the Laplace transforms are expressed in terms $\alpha$

$$
\mathrm{L}\left(\mathrm{y}_{\mathrm{i}}(\mathrm{x})\right)=\int_{0}^{\infty} \mathrm{y}_{\mathrm{i}}(\mathrm{x}) \mathrm{e}^{-\alpha \mathrm{x}} \mathrm{dx}=\mu_{\mathrm{i}}(\alpha) \mathrm{i}=1, \ldots, \mathrm{k}
$$

The problem lies in finding the anti-transformation of $\mu_{i}(\alpha) i=$ $1, \ldots, \mathrm{k}$

In this work we propose to find a numerical approximation of the antitransformation of $\mu_{i}(\alpha) i=1, \ldots, k$ using the techniques of 
inverse moments problem. Moreover, the Laplace transform is generalized into a more general operator defined over an interval $(\mathrm{a}, \mathrm{b})$, with Cauchy conditions in a and $\mathrm{b}$. The objective of this work is to show that we can solve the problem using the techniques of inverse moments problem. We focus the study on the numerical approximation.

\section{Inverse moments problem}

The problem of generalized moments [5], [6] is to find a function $\mathrm{f}(\mathrm{x})$ about a domain $\Omega \subset \mathrm{R}^{\mathrm{d}}$ that satisfies the sequence of equations

$$
\mu_{\mathrm{i}}=\int_{\Omega} g_{\mathrm{i}}(\mathrm{x}) \mathrm{f}(\mathrm{x}) \mathrm{dx} \mathrm{i} \in \mathrm{N}
$$

Where $\mathrm{N}$ is the set of the natural numbers, $\left(\mathrm{g}_{\mathrm{i}}\right)$ is a given sequence of functions in $L^{2}(\Omega)$ linearly independent known and the succession of real numbers $\left\{\mu_{\mathrm{i}}\right\}_{\mathrm{i} \in \mathrm{N}}$ are known data.

The Hausdorff moments problem [6], [7]) is a classic example of a moments problem, we must find a function $f(x)$ in $(a, b)$ such that

$\mu_{i}=\int_{a}^{b} x^{i} f(x) d x i \in N$

In this case $g_{i}(x)=x^{i}$ with $i$ in $N$.

If the integration interval is $(0, \infty)$ we have the moments problem of Stieltjes; if the integration interval is $(-\infty, \infty)$ we have the moments problem of Hamburger [6, 7].

The moments problem is a badly conditioned problem in the sense that there may be no solution and if there is solution, there are not continuous dependence on the given data $[5,6,7]$. There are several methods to build regularized solutions. One of them is the truncated expansion method [5].

This method involves solving (3) considering the finite moments problem

$$
\mu_{i}=\int_{\Omega} g_{i}(x) f(x) d x i=1,2, \ldots, n,
$$

Where the approximate solution of $\mathrm{f}(\mathrm{x})$ is $\mathrm{p}_{\mathrm{n}}(\mathrm{x})=\sum_{\mathrm{i}=1}^{\mathrm{n}} \lambda_{\mathrm{i}} \varphi_{\mathrm{i}}(\mathrm{x})$ and the functions $\varphi_{\mathrm{i}}(\mathrm{x})$ result of orthonormalize $\mathrm{g}_{1}, \mathrm{~g}_{2}, \ldots, \mathrm{g}_{\mathrm{n}}$ and $\lambda_{\mathrm{i}}$ are coefficients that depend on the data $\mu_{\mathrm{i}}$. In the subspace generated by $g_{1}, g_{2}, \ldots, g_{n}$ the solution is stable. If $n \in N$ is chosen in an appropriate way then the solution of (4) is close to solving the original problem (3).

In the case where the data $\mu_{1}, \mu_{2}, \ldots, \mu_{\mathrm{n}}$ are inaccurate, convergence theorems and error estimates must be applied for the regularized solution (pág. 19 a 30 de [5]).

Another method is the Tikhonov method (pág. 18 de [5]). In this method you write (3) in the way Af $=\mu$ with

$\mathrm{Af}=\left(\int_{\Omega} \mathrm{g}_{1} \mathrm{f}, \int_{\Omega} \mathrm{g}_{2} \mathrm{f}, \ldots\right), \mu=\left(\mu_{1}, \mu_{2}, \ldots\right)$

And we must find $\mathrm{f} \epsilon \mathrm{L}^{2}(\Omega)$ that satisfies the variational equation

$\beta(\mathrm{f}, \mathrm{v})_{\mathrm{L}^{2}(\Omega)}+(\mathrm{Af}, \mathrm{Av})_{\mathrm{l}^{2}}=(\mathrm{f}, \mathrm{Av})_{\mathrm{l}^{2}}, \forall \mathrm{v} \in \mathrm{L}^{2}(\Omega)$,

where $(., .)_{\mathrm{L}^{2}(\Omega)}$ and $(., .)_{\mathrm{l}^{2}}$ are the usual internal products of $\mathrm{L}^{2}(\Omega)$ and $\mathrm{l}^{2}$ respectively and $\beta>0$.

\section{Systems of lineal ordinary differential equations}

Given a system of ordinary linear differential equations with constant coefficients of the form $y_{1}^{(n)}(x)=f_{1}\left(x, y_{1}, y_{2}, \ldots, y_{k}, y_{1}^{(1)}, \ldots, y_{k}^{(1)}, \ldots, y_{1}^{(n-1)}, \ldots, y_{k}^{(n-1)}\right)$
$\vdots$
$y_{k}^{(n)}(x)=f_{k}\left(x, y_{1}, y_{2}, \ldots, y_{k}, y_{1}^{(1)}, \ldots, y_{k}^{(1)}, \ldots, y_{1}^{(n-1)}, \ldots, y_{k}^{(n-1)}\right)$

Where $y_{i}^{(n)}(x)$ indicates the derivative of order $n$ of $y_{i}(x) i=$ $1, \ldots, \mathrm{k}$ and

$f_{i}\left(x, y_{1}, y_{2}, \ldots, y_{k}, y_{1}^{(1)}, \ldots, y_{k}^{(1)}, \ldots, y_{1}^{(n-1)}, \ldots, y_{k}^{(n-1)}\right)$

$\mathrm{i}=1, \ldots, \mathrm{k}$

Are linear functions of

$\mathrm{x}, \mathrm{y}_{1}(\mathrm{x}), \mathrm{y}_{2}(\mathrm{x}), \ldots, \mathrm{y}_{\mathrm{k}}(\mathrm{x})$,

$y_{1}^{(1)}(x), \ldots, y_{k}^{(1)}(x), \ldots, y_{1}^{(n-1)}(x), \ldots, y_{k}^{(n-1)}(x)$

With constant coefficients, we want to find numerical approximations for functions $\mathrm{y}_{1}(\mathrm{x}), \mathrm{y}_{2}(\mathrm{x}), \ldots, \mathrm{y}_{\mathrm{k}}(\mathrm{x})$ which are solution of the given system. We assume Cauchy conditions in an interval $(a, b)$

$\mathrm{y}_{1}(\mathrm{a}), \mathrm{y}_{1}^{(1)}(\mathrm{a}), \ldots \ldots \ldots, \mathrm{y}_{1}^{(\mathrm{n}-1)}(\mathrm{a})$

$y_{k}(a), y_{k}^{(1)}(a), \ldots \ldots \ldots, y_{k}^{(n-1)}(a)$

$\mathrm{y}_{1}(\mathrm{~b}), \mathrm{y}_{1}^{(1)}(\mathrm{b}), \ldots \ldots \ldots, \mathrm{y}_{1}^{(\mathrm{n}-1)}(\mathrm{b})$

$y_{k}(b), y_{k}^{(1)}(b), \ldots \ldots \ldots, y_{k}^{(n-1)}(b)$

We also assume that each $\mathrm{y}_{\mathrm{i}}(\mathrm{x}) \in \mathrm{L}^{2}(\mathrm{a}, \mathrm{b})$.

We define the operator

$L^{*}(y(x))=\int_{a}^{b} y(x) e^{-\alpha x} d x$

Integrating (7) by parts you get to the relationship

$\mathrm{L}^{*}\left(\mathrm{y}^{(1)}(\mathrm{x})\right)=\mathrm{y}(\mathrm{b}) \mathrm{e}^{-\alpha \mathrm{b}}-\mathrm{y}(\mathrm{a}) \mathrm{e}^{-\alpha \mathrm{a}}+\alpha \mathrm{L}^{*}(\mathrm{y}(\mathrm{x}))$

Integrating by parts (7) repeatedly we come to

$\mathrm{L}^{*}\left(\mathrm{y}^{(\mathrm{n})}(\mathrm{x})\right)=$

$=\mathrm{e}^{-\alpha \mathrm{b}}\left[\mathrm{y}^{(\mathrm{n}-1)}(\mathrm{b})+\alpha \mathrm{y}^{(\mathrm{n}-2)}(\mathrm{b})+\cdots+\alpha^{\mathrm{n}-1} \mathrm{y}(\mathrm{b})\right]-$

$-e^{-\alpha a}\left[y^{(n-1)}(a)+\alpha y^{(n-2)}(a)+\cdots+\alpha^{n-1} y(a)\right]++\alpha^{n} L^{*}(y(x))$

Note that if in (7) $\mathrm{b} \rightarrow \infty$, and $\mathrm{a}=0$ then it becomes the property of the Laplace transform named above.

We apply $\mathrm{L}^{*}$ to each system equation (5) and taking into account (8) we arrive at a system of algebraic equations

$\begin{array}{ccc}a_{11}(\alpha) L^{*}\left(y_{1}\right)+a_{12}(\alpha) L^{*}\left(y_{2}\right)+ & \ldots & +a_{1 k}(\alpha) L^{*}\left(y_{k}\right)=c_{1}(\alpha) \\ \vdots & \ldots & \vdots \\ a_{k 1}(\alpha) L^{*}\left(y_{1}\right)+a_{k 2}(\alpha) L^{*}\left(y_{2}\right)+ & \ldots & +a_{k k}(\alpha) L^{*}\left(y_{k}\right)=c_{k}(\alpha)\end{array}$

Where the unknowns are $L^{*}\left(y_{1}\right), L^{*}\left(y_{2}\right), \ldots, L^{*}\left(y_{k}\right)$.

When solving the system (9), the unknowns $L^{*}\left(y_{i}\right) i=1, \ldots, k$ are expressed in terms of $\alpha$, that is to say $L^{*}\left(y_{i}\right)=\mu_{i}(\alpha) i=1, \ldots, k$. We write A to the matrix of the coefficients

$\mathrm{A}=\left(\begin{array}{ccc}\mathrm{a}_{11}(\alpha) & \mathrm{a}_{12}(\alpha) \ldots & \mathrm{a}_{1 \mathrm{k}}(\alpha) \\ \vdots & \vdots & \vdots \\ \mathrm{a}_{\mathrm{k} 1}(\alpha) & \mathrm{a}_{\mathrm{k} 2}(\alpha) \ldots & +\mathrm{a}_{\mathrm{kk}}(\alpha)\end{array}\right)$

If $\operatorname{Det}(A) \neq 0$ then the system (9) have unique solution. We change the variable $\mathrm{z}=\mathrm{e}^{-\mathrm{x}}$ and we get 
$\mathrm{L}^{*}\left(\mathrm{y}_{\mathrm{i}}\right)=$

$=\int_{a}^{b} y_{i}(x) e^{-\alpha x} d x=\int_{e^{-b}}^{e^{-a}} y_{i}(-\ln (z)) z^{\alpha-1} d z=\int_{a_{1}}^{b_{1}} y_{i}^{*}(z) z^{\alpha-1} d z$

Where $a_{1}=e^{-b} ; b_{1}=e^{-a}$ and $y_{i}^{*}(z)=y_{i}(-\ln (z))$.

Then we can interpret

$$
\int_{a_{1}}^{b_{1}} y_{i}^{*}(z) z^{\alpha-1} d z=\mu_{i}(\alpha)
$$

As a inverse moments problem giving $\alpha$ values such that $\operatorname{Det}(A) \neq 0$.

The moments problem is solved considering the corresponding moments problem finite, that is, assigning to $\alpha$ a finite number of values, $\alpha=$ alf $a, \ldots, n$, with alfa chosen conveniently so that $\operatorname{Det}(A) \neq 0$.

This is repeated in each $L^{*}\left(y_{i}\right)=\mu_{i}(\alpha) i=1, \ldots, k$. To apply the truncated expansion method, write $(10)$ as

$\int_{a_{1}}^{b_{1}} y_{i}^{*}(z) z^{\alpha l f a-1} z^{\alpha-a l f a} d z=\mu_{i}(\alpha)$

We get an approximate solution $p_{n i}(z)$ for each $y_{i}^{*}(z) z^{\alpha l f a-1}$. Then the approximate solution for $y_{i}(x)$ be $y_{i}(x) \approx$ $\left(e^{x}\right)^{\text {alf } a-1} p_{n i}\left(e^{-x}\right)$.

In the case of being $(a, b)$ an unbounded interval, for example $(a, \infty)$, it is convenient to proceed in another way, without changing the variable because in certain cases the norm $L^{2}$ of the difference $y_{i}(x)-\left(e^{x}\right)^{a l f a-1} p_{n i}\left(e^{-x}\right)$ it would be divergent.

This second procedure [8] consists of taking a base $\left\{\psi_{r}(\alpha)\right\}_{r}$ to $L^{2}(a, \infty)$ and then

$\int_{a}^{\infty} y_{i}(x) e^{-\alpha x} d x=\mu_{i}(\alpha)$

Can be transformed into a generalized problem of moments by multiplying both members of equality by $\psi_{r}(\alpha)$ and integrate with respect to $\alpha$. In this way we come to

$\int_{a}^{\infty} y_{i}(x) g_{r}(x) d x=\mu_{i r} r=1,2, \ldots$

Where

$g_{r}(x)=\int_{a}^{\infty} e^{-\alpha x} \psi_{r}(\alpha) d \alpha$

And the moments $\mu_{i r}$ are

$\mu_{i r}=\int_{a}^{\infty} \mu_{i}(\alpha) \psi_{r}(\alpha) d \alpha$

This procedure can also be applied if $(\mathrm{a}, \mathrm{b})$ is a finite interval.

\section{Solution of inverse moments problem}

To solve (10) numerically as a moments problem, we apply the truncated expansion method detailed in [7], and generalized in [8], in order to find an approximation $p_{n i}(z)$ from $y_{i}^{*}(z) z^{\alpha l f a-1}$ for the corresponding finite problem with $\alpha=\operatorname{alf} a, \ldots, n$; where $\mathrm{n}$ is the number of moments $\mu_{i}(\alpha)$ that are considered.

Let $\phi_{\alpha}(z) \alpha=$ alfa, $\ldots, n$ the base obtained by orthonormalizing $z^{\alpha-a l f a} ; \alpha=$ alfa, $\ldots, n$ and adding to the resulting set the necessary functions until reaching an orthonormal basis.

Or, written in another way, $z^{r} ; r=0, \ldots, n^{*} n^{*}=n-$ alf $a+1$ If the interval is unbounded, the functions $g_{r}(x)$ previously defined are orthonormalized.

The function $y_{i}^{*}(z)$ is approximated by the truncated expansion method:

$$
p_{n i}(z)=\sum_{r=0}^{n^{*}} \lambda_{r} \phi_{r}(z) \quad ; \quad \lambda_{r}=\sum_{j=0}^{r} C_{r j} \mu_{j} \quad r=0,1, \ldots ., n^{*},
$$

And $C_{r j}$ are the coefficients of a matrix $C$ that verify

$C_{r j}=\left(\sum_{k=j}^{r-1}(-1) \frac{\left\langle z^{r} \mid \phi_{k}(z)\right\rangle}{\left\|\phi_{k}(z)\right\|^{2}} C k j\right)\left\|\phi_{\phi_{r}(z)}\right\|^{-1} \quad 1<r \leq n ; 1 \leq j<r$,

The terms of the diagonal are

$$
C_{r r}=\left\|\phi_{\phi_{r}}(z)\right\|^{-1} \quad r=0,1, \ldots, n . * *
$$

The following theorem gives a measure of the accuracy of the approximation.

Theorem: Let $\left\{\mu_{r}\right\}_{r=0}^{n^{*}}$ be set of real numbers and suppose that $y(z)$ in $L^{2}\left(a_{1}, b_{1}\right)$ verify for some $n^{*}, \varepsilon y M$ (two positive numbers):

$\sum_{r=0}^{n^{*}}\left|\int_{a_{1}}^{b_{1}} z^{r} y(z) d z-\mu_{r}\right|^{2} \leq \varepsilon^{2}$

And

$\int_{a_{1}}^{b_{1}}\left|y^{(1)}(z)\right|^{2} d z \leq M^{2}$

Then

$$
\int_{a_{1}}^{b_{1}}\left|y(z)-p_{n^{*}}(z)\right|^{2} d z \leq\left\|C^{T} C\right\| \varepsilon^{2}+\frac{\left(b_{1}-a_{1}\right)^{2}}{4\left(n^{*}+1\right)^{2}} M^{2}
$$

If the interval is $(a, \infty)$, then the condition (11) change by

$\int_{a}^{\infty} z e^{z}\left(y^{(1)}(z)\right)^{2} d z \leq M^{2}$

And the conclusion (12) change by

$\int_{a}^{\infty}\left|y(z)-p_{n^{*}}(z)\right|^{2} d z \leq\left\|C^{T} C\right\| \varepsilon^{2}+\frac{M^{2}}{\left(n^{*}+1\right)^{2}}$

In addition, it must be fulfilled that

$z^{r} y(z) \rightarrow 0$ if $z \rightarrow \infty$ for all $r \in N$.:

The proof of this Theorem is detailed in [8] for the case of bounded interval, and in [9] for the interval case $\left(a_{1}, \infty\right)$.

\section{Numerical examples}

We illustrate the above with simple examples.

\subsection{Example 1}

We considered the system of equations

$\left\{\begin{array}{c}y^{(2)}(x)+2 y(x)+4 z(x)=e^{x} \\ z^{(2)}(x)-y(s)-3 z(x)=-x\end{array}\right.$

In the interval $(1,3)$ under the conditions

$\left\{\begin{array}{c}y(1)=-2+e+e^{-\sqrt{2}}+e^{\sqrt{2}}+\cos (1)+\operatorname{sen}(1) \\ y(3)=-6+e^{3}+e^{-3 \sqrt{2}}+e^{3 \sqrt{2}}+\cos (3)+\operatorname{sen}(3)\end{array}\right.$

$\left\{\begin{array}{c}z(1)=1-\frac{e}{2}-e^{-\sqrt{2}}-e^{\sqrt{2}}-\frac{\cos (1)}{4}-\frac{\operatorname{sen}(1)}{4} \\ z(3)=3+\frac{e^{3}}{2}-e^{-3 \sqrt{2}}-e^{3 \sqrt{2}}-\frac{\cos (3)}{4}-\frac{\operatorname{sen}(3)}{4}\end{array}\right.$

$\left\{y^{(1)}(1)=-2+e-\sqrt{2} e^{-\sqrt{2}}+\sqrt{2} e^{\sqrt{2}}+\cos (1)-\operatorname{sen}(1)\right.$ 


$$
\left\{\begin{array}{c}
z^{(1)}(1)=1-\frac{e}{2}+\sqrt{2} e^{-\sqrt{2}}-\sqrt{2} e^{\sqrt{2}}-\frac{\cos (1)}{4}+\frac{\operatorname{sen}(1)}{4} \\
z^{(1)}(3)=1-\frac{e^{3}}{2}+\sqrt{2} e^{-3 \sqrt{2}}-\sqrt{2} e^{3 \sqrt{2}}-\frac{\cos (3)}{4}+\frac{\operatorname{sen}(3)}{4}
\end{array}\right.
$$

The exact solution of the system is

$$
\left\{\begin{array}{c}
y(x)=e^{x \sqrt{2}}+e^{-x \sqrt{2}}+\operatorname{sen}(x)+\cos (x)+e^{x}-2 x \\
z(x)=-e^{x \sqrt{2}}-e^{-x \sqrt{2}}-\frac{1}{4} \operatorname{sen}(x)-\frac{1}{4} \cos (x)-\frac{e^{x}}{2}+x
\end{array}\right.
$$

We apply the operator $L^{*}$ to both system equations and you get to

$$
\left\{\begin{array}{l}
\left(\alpha^{2}+2\right) L^{*}(y)+4 L^{*}(z)=L^{*}\left(e^{x}\right)-c y(\alpha) \\
-L^{*}(y)+\left(\alpha^{2}-3\right) L^{*}(z)=L^{*}(-x)-c z(\alpha)
\end{array}\right.
$$

Where $c y(\alpha)$ and $c z(\alpha)$ are expressions depending on the conditions (13), (14), (15) y (16)

The determinant of the matrix of system coefficients (17) is

$\left|\begin{array}{cc}\alpha^{2}+2 & 4 \\ -1 & \alpha^{2}-3\end{array}\right|=\alpha^{4}-\alpha^{2}-2$

And it is null for $=-i, \alpha=i, \alpha=\sqrt{2}, \alpha=-\sqrt{2}$.

We solve the system with Mathematica software and get expressions for $L^{*}(y)$ y $L^{*}(z)$ as a function of $\alpha$.

We evaluate these expressions giving values to $\alpha$ since alfa $a=2$ until $n=8$, that is, we take 7 "moments" $\mu(\alpha)$.

The value for alfa it is set equal to 2 in order to avoid discontinuities.

Applying the truncated expansion method we obtain an approximation for $y(x)$ given by

$y(x) \approx\left(e^{x}\right)^{a l f a-1} p y_{n}\left(e^{-x}\right)$

Whose accuracy is

$\int_{1}^{3}\left|y(x)-\left(e^{x}\right)^{a l f a-1} p y_{n}\left(e^{-x}\right)\right|^{2} d x=0.0557505$

Analogously, for $z(x)$ we obtain an accuracy of

$$
\int_{1}^{3}\left|z(x)-\left(e^{x}\right)^{a l f a-1} p z_{n}\left(e^{-x}\right)\right|^{2} d x=0.0543516
$$

In the Fig. 1 and in the Fig. 2 we observe the graphs of $y(x)$ and $z(x)$ with their respective overlapping approximations.

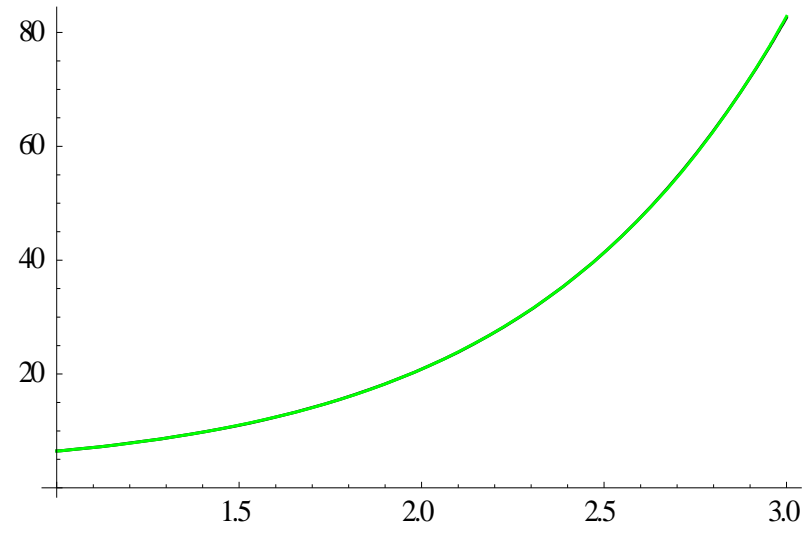

Fig. 1: $\mathrm{Y}(\mathrm{X})$ And Its Approach.

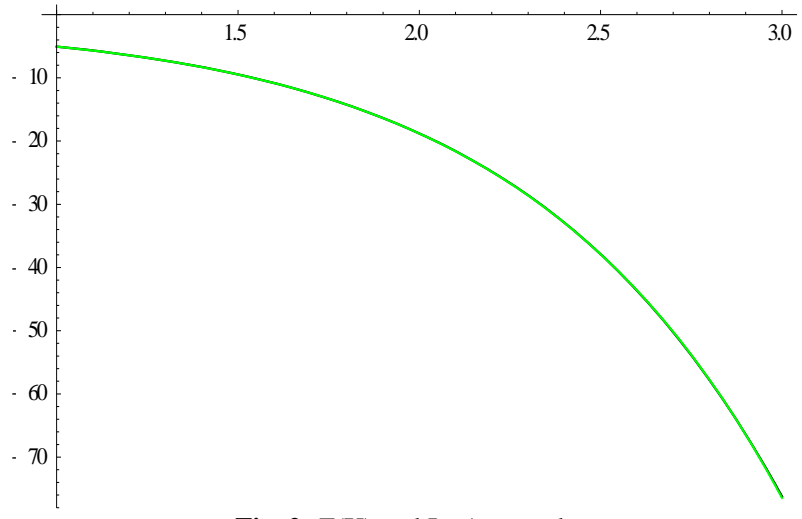

Fig. 2: $\mathrm{Z}(\mathrm{X})$ and Its Approach

\subsection{Example 2}

We considered the system of equations

$\left\{\begin{array}{c}y^{(1)}(x)=3 z(x)-4 u(x) \\ z^{(1)}(x)=-u(x) \\ u^{(1)}(x)=z(x)-2 y(x)\end{array}\right.$

In the interval $(0,1)$ under the conditions

$\left\{\begin{array}{c}y(0)=3 ; y(1)=e^{3}+\frac{1}{e^{2}}+\frac{1}{e} \\ z(0)=1.6 ; z(1)=4.43912 \\ u(0)=1.2 ; u(1)=-11.5752\end{array}\right.$

The exact solution of the system is

$\left\{\begin{array}{c}y(x)=e^{-x}+e^{-2 x}+e^{3 x} \\ z(x)=e^{-x}+0.4 e^{-2 x}+0.2 e^{3 x} \\ u(x)=e^{-x}+0.8 e^{-2 x}-0.6 e^{3 x}\end{array}\right.$

We apply the operator $L^{*}$ to both system equations and you get to

$\left\{\begin{array}{c}\alpha L^{*}(y)-3 L^{*}(z)+4 L^{*}(u)=-c y(\alpha) \\ \alpha L^{*}(z)+L^{*}(u)-c z(\alpha) \\ 2 L^{*}(y)-L^{*}(z)+\alpha L^{*}(u)=-c u(\alpha)\end{array}\right.$

Where $c y(\alpha), c z(\alpha)$ and $c u(\alpha)$ are expressions depending on the conditions (18).

The determinant of the matrix of system coefficients (19) is

$\left|\begin{array}{ccc}\alpha & -3 & 4 \\ 0 & \alpha & 1 \\ 2 & -1 & \alpha\end{array}\right|=\alpha^{3}-7 \alpha-6$

And it is null for $\alpha=-2 ; \alpha=-1 ; \alpha=3$.

We solve the system with Mathematica software and get expressions for $L^{*}(y), L^{*}(z) y L^{*}(u)$ as a function of $\alpha$.

We apply the first procedure to solve the system by changing the variable, since when trying to apply the second procedure we find a discontinuity when orthonormalizing the base.

We evaluate these expressions giving values to $\alpha$ since alf $a=4$ until $n=8$, that is, we take 5 "moments" $\mu(\alpha)$.

The value for alfa it is set equal to 4 in order to avoid discontinuities and for the solution to be unique.

Applying the truncated expansion method we obtain an approximation for $y(x)$ given by

$\left(e^{x}\right)^{a l f a-1} p y_{n}\left(e^{-x}\right)$

Whose accuracy is

$\int_{0}^{1}\left|y(x)-\left(e^{x}\right)^{a l f a-1} p y_{n}\left(e^{-x}\right)\right|^{2} d x=0.00135636$ 
In the Fig. 3 we observe the graphs of $y(x)$ with their overlapping approximation.

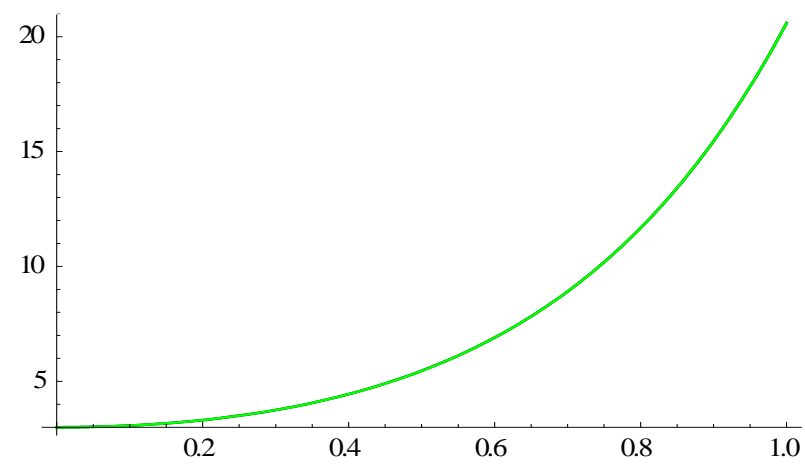

Fig. 3: $\mathrm{Y}(\mathrm{X})$ and its Approach.

Analogously, for $z(x)$ we obtain an accuracy of

$\int_{0}^{1}\left|z(x)-\left(e^{x}\right)^{a l f a-1} p z_{n}\left(e^{-x}\right)\right|^{2} d x=0.000542543$

In the Fig. 4 we observe the graphs of $z(x)$ with their overlapping approximation.

Finally we get the approximation for $u(x)$. In this case we have an accuracy of

$\int_{0}^{1}\left|u(x)-\left(e^{x}\right)^{a l f a-1} p u_{n}\left(e^{-x}\right)\right|^{2} d x=0.00108509$

In the Fig. 5 we observe the graphs of $u(x)$ with their overlapping approximation.

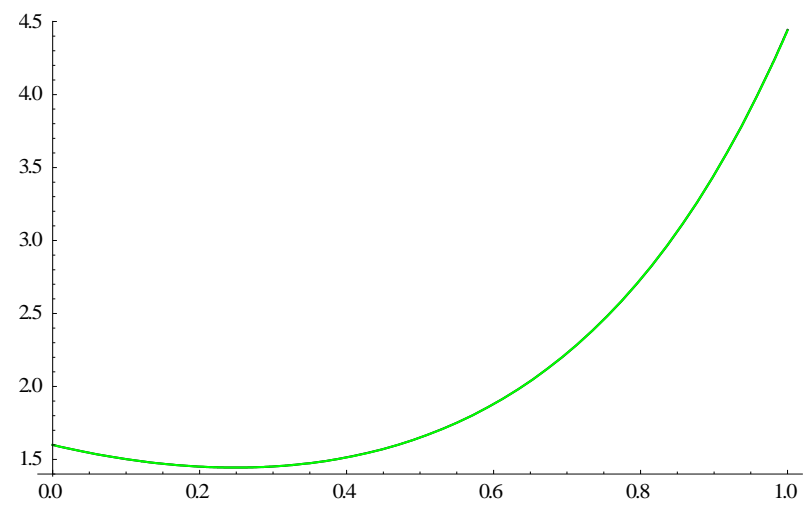

Fig. 4: $\mathrm{Z}(\mathrm{X})$ and its Approach.

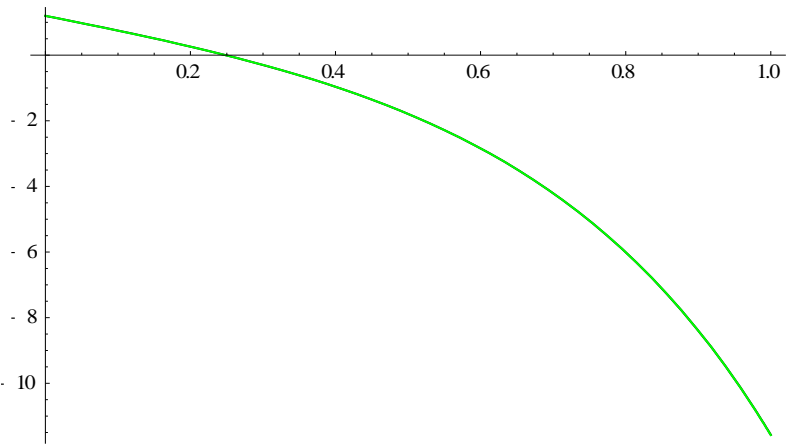

Fig. 5: $\mathrm{U}(\mathrm{X})$ and its Approach.

\subsection{Example 3}

We considered the system of equations

$$
\left\{\begin{array}{c}
y^{(1)}(x)+3 y(x)+z(x)=0 \\
z^{(1)}(x)-y(x)+z(x)=0
\end{array}\right.
$$

In the interval $(0, \infty)$ under the conditions $y(0)=1 ; z(0)=-2$

The exact solution of the system is

$\left\{\begin{array}{l}y(x)=(1+x) e^{-2 x} \\ z(x)=-(2+x) e^{-2 x}\end{array}\right.$

We apply the Laplace transform to both system equations (20) and we come to

$$
\left\{\begin{array}{c}
(\alpha+3) L(y)+L(z)=1 \\
-L(y)+(\alpha+1) L(z)=-2
\end{array}\right.
$$

The determinant of the matrix of system coefficients (21) is

$\left|\begin{array}{cc}3+\alpha & 1 \\ -1 & 1+t\end{array}\right|=\alpha^{2}+4 \alpha+4$

In addition, it is null for $\alpha=-2$

We solve the system with Mathematica software and get expressions for $L(y)$ y $L(z)$ as a function of $\alpha$ :

$\left\{\begin{array}{l}L(y)=\frac{3+\alpha}{(2+\alpha)^{2}} \\ L(z)=\frac{-5-2 \alpha}{(2+\alpha)^{2}}\end{array}\right.$

We consider the basis $\left\{\psi_{r}(\alpha)\right\}_{r}=\left\{\alpha^{r} e^{-\alpha}\right\}_{\alpha}$ of $L^{2}(0, \infty)$. We apply the second procedure for $n=4$ moments.

With the truncated expansion method, we obtain an approximation for $y(x)$ whose accuracy is

$\int_{0}^{\infty}\left|y(x)-p y_{n}(x)\right|^{2} d x=0.0203557$

Analogously, for $z(x)$ we obtain an accuracy of

$\int_{0}^{\infty}\left|z(x)-p z_{n}(x)\right|^{2} d x=0.02963743$.

In the Fig. 6 we observe the graphs of $y(x)$ with their overlapping approximation.

Analogously in the Fig. 7 for $z(x)$ and its approach.

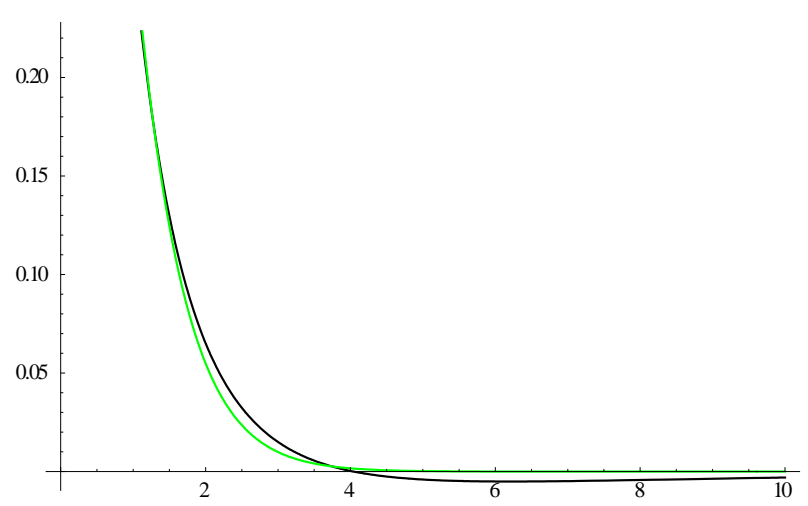

Fig. 6: $\mathrm{Y}(\mathrm{X})$ and its Approach

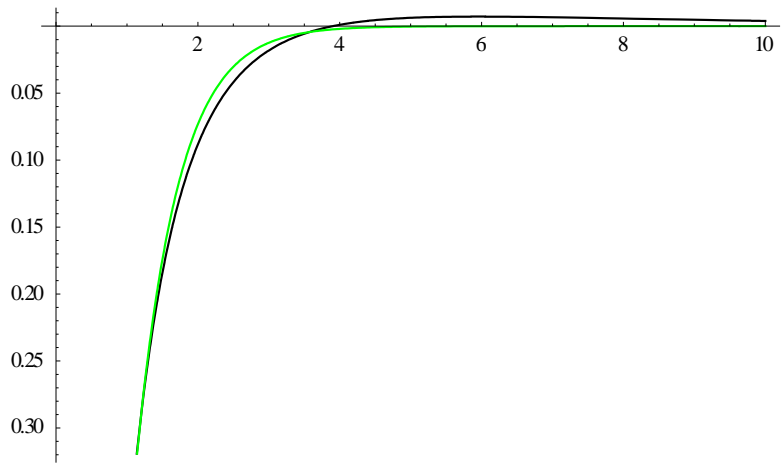

Fig. 7: $\mathrm{Z}(\mathrm{X})$ and its Approach. 


\section{Conclusion}

Given a system of ordinary linear differential equations with constant coefficients of the form

$y_{1}^{(n)}(x)=f_{1}\left(x, y_{1}, y_{2}, \ldots, y_{k}, y_{1}^{(1)}, \ldots, y_{k}^{(1)}, \ldots, y_{1}^{(n-1)}, \ldots, y_{k}^{(n-1)}\right)$

$y_{k}^{(n)}(x)=f_{k}\left(x, y_{1}, y_{2}, \ldots, y_{k}, y_{1}^{(1)}, \ldots, y_{k}^{(1)}, \ldots, y_{1}^{(n-1)}, \ldots, y_{k}^{(n-1)}\right)$

Where

$y_{i}^{(n)}(x)$ indicates the derivative of order $\mathrm{n}$ of $y_{i}(x)$ with $i=$ $1, \ldots, k$, and

$f_{i}\left(x, y_{1}, y_{2}, \ldots, y_{k}, y_{1}^{(1)}, \ldots, y_{k}^{(1)}, \ldots, y_{1}^{(n-1)}, \ldots, y_{k}^{(n-1)}\right)$

$i=1, \ldots, k$ Are linear functions of?

$$
x, \mathrm{y}_{1}(\mathrm{x}), \mathrm{y}_{2}(\mathrm{x}), \ldots, \mathrm{y}_{\mathrm{k}}(\mathrm{x}) \mathrm{y}_{1}^{(1)}(\mathrm{x}), \ldots, \mathrm{y}_{\mathrm{k}}^{(1)}(\mathrm{x}), \ldots, \mathrm{y}_{1}^{(\mathrm{n}-1)}(\mathrm{x}), \ldots, \mathrm{y}_{\mathrm{k}}^{(\mathrm{n}-1)}(\mathrm{x})
$$

With constant coefficients, can be found in approximate form, the functions $\mathrm{y}_{1}(\mathrm{x}), \mathrm{y}_{2}(\mathrm{x}), \ldots, \mathrm{y}_{\mathrm{k}}(\mathrm{x})$, which are solution of the given system, under Cauchy conditions in an interval $(a, b)$, considering the operator

$$
L^{*}(y(x))=\int_{a}^{b} y(x) e^{-\alpha x} d x
$$

Which coincides with the Laplace Transform if $\mathrm{a}=0 \mathrm{andb}=\infty$. We also assume that eachy $(x) \in L^{2}(a, b)$.

When applying said operator on each equation of the system, a system of algebraic equations is obtained where the unknowns are $L^{*}\left(y_{1}\right), L^{*}\left(y_{2}\right), \ldots, L^{*}\left(y_{k}\right)$ and the coefficients are given by expressions depending on $\alpha$.

Therefore, when solving the system of algebraic equations the unknowns are equated to expressions in function of $\alpha$

$$
\int_{a}^{b} y_{i}(x) e^{-\alpha x} d x=\mu_{i}(\alpha)
$$

Making change of variable and discretizing the problem, giving to $\alpha$ appropriate value, it can be interpreted as a inverse moments problem and solved using the techniques of truncated expansion. In that, way we obtain a numerical approximation for eachy $\mathrm{y}_{\mathrm{i}}(\mathrm{x})$. In the case of having an interval of the form $(a, \infty)$, we multiply both members of the previous equality by a base of $\mathrm{L}^{2}(a, \infty)$ and we integrate. In this way, we obtain equality

$$
\int_{a}^{\infty} y_{i}(x) g_{r}(x) d x=\mu_{i r} r=1,2, \ldots
$$

Where

$g_{r}(x)=\int_{a}^{\infty} e^{-\alpha x} \psi_{r}(\alpha) d \alpha$

In addition, the moments $\mu_{\mathrm{r}}$ are

$\mu_{\mathrm{ir}}=\int_{\mathrm{a}}^{\infty} \mu_{\mathrm{i}}(\alpha) \psi_{\mathrm{r}}(\alpha) \mathrm{d} \alpha$

This procedure can also be applied if $(\mathrm{a}, \mathrm{b})$ is a finite interval. Again applying the truncated expansion method to the corresponding moments problem, we find a numerical approximation for each $y_{i}(x)$.

\section{Acknowledgement}

My thanks to the Faculty of Engineering and to the Faculty of Exact Sciences of the National University of La Plata, Argentina.

\section{References}

[1] John H. Hubbard, Beverly H. West, Differential Equations: A Dy namical Systems Approach, Springer-Verlag, New York, Inc. 1995

[2] David Kincaid, Ward Cheney, Análisis Numérico, las matemáticas Del cálculo científico, Addison-Wesley Iberoamericana, 1994.

[3] Rubio Sanjuan, Ampliación de Matemáticas, Editorial Labor, 1951

[4] Charles E. Roberts, Ordinary Differential Equations, CRC Press Taylor \& Francis Group, 2018

[5] D.D. Ang, R. Gorenflo, V.K. Le and D.D. Trong, Moment theory and some inverse problems in potential theory and heat conduction, Lectures Notes in Mathematics, Springer-Verlag, Berlin, 2002. https://doi.org/10.1007/b84019.

[6] J.A. Shohat and J.D. Tamarkin, The problem of Moments, Mathematic Surveys, Am. Math. Soc., Providence, RI, 1943. https://doi.org/10.1090/surv/001.

[7] G. Talenti, "Recovering a function from a finite number of moments", Inverse Problems 3, (1987), pp.501- 517. https://doi.org/10.1088/0266-5611/3/3/016.

[8] M. B. Pintarelli and F. Vericat, "Stability theorem and inversion algorithm for a generalize moment problem", Far East Journal of Mathematical Sciences, 30, (2008), pp. 253-274.

[9] M. B. Pintarelli, "Parabolic Partial Differential Equations as Inverse Moments Problem", Applied Mathematics, Vol7 Number1, (2016), pp. 77-99. 